\title{
Local treatment of synchronous oligometastatic non-small cell lung cancer (NSCLC)-current consensus and future perspectives
}

\author{
Doaa Said Almeldin ${ }^{1,2}$, Jyoti Malhotra ${ }^{3}$, Malini Patel ${ }^{3}$, Joseph Aisner ${ }^{3}$, Salma K. Jabbour ${ }^{1}$ \\ ${ }^{1}$ Department of Radiation Oncology, Rutgers Cancer Institute of New Jersey, Robert Wood Johnson Medical School, Rutgers University, New \\ Brunswick, NJ, USA; ${ }^{2}$ Clinical Oncology Department, Cairo University, Cairo, Egypt; ${ }^{3}$ Division of Medical Oncology, Rutgers Cancer Institute of \\ New Jersey, Robert Wood Johnson Medical School, Rutgers University, New Brunswick, NJ, USA \\ Correspondence to: Salma K. Jabbour, MD. Department of Radiation Oncology, Rutgers Cancer Institute of New Jersey, 195 Little Albany Street, New \\ Brunswick, NJ 08903, USA. Email: jabbousk@cinj.rutgers.edu. \\ Comment on: Dingemans AC, Hendriks LEL, Berghmans T, et al. Definition of Synchronous Oligometastatic Non-Small Cell Lung Cancer-A \\ Consensus Report. J Thorac Oncol 2019;14:2109-19.
}

Submitted Mar 25, 2020. Accepted for publication May 06, 2020.

doi: $10.21037 /$ jtd-20-1485

View this article at: http://dx.doi.org/10.21037/jtd-20-1485

The integration of effective systemic therapies such as immunotherapy with immune checkpoint blockade (ICB) and targeted therapies improves survival outcomes significantly for patients with advanced non-small cell lung cancer (NSCLC). Mounting clinical evidence suggests that the application of radical locoregional therapies (LRT), including surgery and stereotactic body radiotherapy (SBRT) to synchronous oligometastatic NSCLC (SOMNSCLC) improves outcomes beyond those achieved using systemic therapies alone $(1,2)$. However, more precise definitions of SOM-NSCLC are needed to facilitate the care of patients and interpretation of prospective clinical trial results.

Although metastatic NSCLC historically had dismal survival rates, research has explored heterogeneity within this group to determine which patients may benefit from LRT to selected sites of oligometastases. The biological concept of oligometastatic cancer was initially introduced in 1995 by Drs. Hellman and Weichselbaum as an intermediate state of limited tumor foci in one or limited organs that differs from the classic microscopic or "leukemia-like" dissemination (3). They explained differing virulence patterns between SOM and metachronous OM disease; for SOM, limited disease sites are diagnosed simultaneously with the primary uncontrolled, and metachronous oligometastatic or "oligo-recurrences" that appear after initial control of the primary with a measurable disease-free interval (DFI) (4). SOM is expected to have better outcomes if controlled initially compared to metachronous oligometastatic disease that succeeded in escaping previous systemic therapy and evading the immune system. Dr. Weichselbaum and Hellman illustrated that temporal evolution of SOM disease will vary according to several factors e.g., (initial disease site and extent, histology, molecular profile, type of first line systemic therapy and its response) with disease free interval $>36$ months carrying the best prognosis (5).

In the Fournal of Thoracic Oncology in December 2019, Dingemans et al. and the Lung Cancer Group of the European Organization of Research and Treatment of Cancer (EORTC) published a multidisciplinary consensus report for the definition of SOM-NSCLC based on a systematic review and results from expert surveys that were designed for this consensus statement (6). Data were collected from 21 studies published between 1996 to 2017 that included 1,215 patients (7). Based on the systematic review, the definition of SOM applied when five or fewer metastatic lesions were identified in up to three different organs. The consensus group defined SOM-NSCLC stage based on the technical feasibility of radical treatment with acceptable toxicity. There was extensive experts discussion about the value of treating a larger number of lesions (e.g., $>5$ ) if still technically feasible. Survey questions including ten "real-life" cases were addressed to thirty thoracic oncology experts to unify the definition of SOM-NSCLC, given the variety of management paradigms. Following the 
$8^{\text {th }}$ TNM staging system (8), contralateral pulmonary lesions were counted as one metastatic site, while nodules within the same lobe of the lung (T3) and ipsilateral lung nodules (T4) did not count as metastases. Also, mediastinal nodes were considered in the $\mathrm{N}$ descriptor and not as metastatic. According to this consensus statement, all organs could be considered for LRT except where radical treatment was not feasible (serosal membranes, meninges and bone marrow). The impact of site-specific metastases on outcome was controversial and the consensus did not endorse any specific sites (i.e., brain or adrenals) to change the concept of oligometastatic disease. One limitation is that histological and genomic classifications were not considered in this consensus statement.

Advanced diagnostic imaging is the current cornerstone to discriminate SOM-NSCLC from the poly-metastatic stage. To avoid toxicities of unnecessary local treatments, in Dingemans et al. there was complete expert agreement on the value of 18-fluorodeoxyglucose-positron emission tomography with computed tomography (18F-FDG PET/ $\mathrm{CT}$ ) and brain magnetic resonance imaging (MRI) for staging. Pathological confirmation of at least one metastasis was emphasized especially in the setting of solitary lesions. Dedicated imaging and biopsies were recommended if believed to change the treatment course (6).

With the promising survival results for SOM-NSCLC, the EORTC consensus statement on definition of this disease stage is a step toward clearer inclusion criteria for future randomized controlled trials. Also, this consensus raised debatable questions and research points to be discussed further. For example, with the definition of SOMNSCLC based mainly on the feasibility of the LRT comes the possibility of long-term toxicities, which need to be considered in routine clinical practice and discussed with patients and multi-disciplinary teams. Although the toxicity profiles of LRT are well tolerated, LRT combination with ICB and targeted therapies requires additional study.

Furthermore, mediastinal lymph nodes are not considered metastatic sites for SOM-NSCLC in the EORTC consensus; however, high nodal burden carries negative impact on prognosis and specifically requires aggressive interventions $(9,10)$. Definitive thoracic therapy for primary and mediastinal nodes should be attempted using surgery or radiotherapy to improve outcomes with ablating oligometastatic sites (11). Invasive mediastinal staging would be mandated to solidify the treatment plan. It will also add an important prognostic point about extent of mediastinal invasion (12). Hypofractionation and SBRT fractionation schemes have been reported to improve locoregional control of the primary thoracic disease in SOM-NSCLC $(13,14)$. Conventional concurrent chemoradiation therapy could also serve as an effective local control strategy for SOM-NSCLC with high mediastinal nodal burden (15).

Since the influence of specific oligometastatic sites on survival is controversial, relapse patterns of certain sites may affect LRT long term outcomes. For example, brain lesions per se should not exclude patients from LRT trials, and SOM-NSCLC patients have higher rates of cranial failure after systemic therapy and extracranial LRT $(11,16)$. For patients presenting with brain lesions, nearly half of the failures reported after local therapy occurred in the brain outside the irradiated field. Frequent monitoring of the neuraxis may be a reasonable strategy for SOMNSCLC when LRT is adopted (15). There are some clinically useful nomograms that could be used to classify patients of higher risk of relapse included in randomized clinical trials of LRT (17). Brain metastases appear to significantly impact overall prognosis for SOM-NSCLC.

A meta-analysis by Ashworth et al. included 49 studies with a total of 757 OM-NSCLC patients and showed that 5 -year overall survival was $29.4 \%$ for patients in favorable risk group (good performance status, lower intrathoracic disease burden and limited metastatic lesions i.e., $</=5$ lesions). Prognostic factors for improved response to LRT were synchronous lesions and nodal stage. Statistically significant poorer outcomes were observed with nonsurgical management of the primary lung cancer and the presence of brain metastases (18). Other prognostic markers to identify tumors with limited metastatic capacity included: number, size of metastatic lesions and number of involved organs (5). Ashworth et al. defined "four aces" or prognostics factors including young age, patient fitness, slow-growing disease (i.e., metachronous metastases or a long DFI between the original cancer and the metastatic recurrence), and low disease burden (i.e., a smaller number of metastases) (9). Patients who had good response to first line chemotherapy tended to benefit when aggressive local treatment is applied to primary thoracic and oligometastatic sites (19).

From the meta-analysis by Giaj-Levra et al. (7), most of the evidence for the additive value of LRT emanated from multiple retrospective and non-randomized studies. However, better-than-expected survival outcomes were 
Table 1 Summary of the two-randomized phase II trials of SOM-NSCLC

\begin{tabular}{|c|c|c|}
\hline Variable/outcomes & Gomez et al. & lyengar et al. \\
\hline Systemic therapy & $\begin{array}{l}\text { Platinum-based doublet or EGFR/ALK targeted } \\
\text { TKI }\end{array}$ & $\begin{array}{l}\text { Platinum-based doublet-EGFR mutations and ALK gene } \\
\text { rearrangement were excluded }\end{array}$ \\
\hline No. of metastatic lesions & $\leq 3$ & $\leq 5$ \\
\hline Primary disease management & $\begin{array}{l}\text { - Intermediate hypofractionation } \\
\text { - Conventional with chemotherapy } \\
\text { - Surgery } \\
\text { - SBRT }\end{array}$ & $\begin{array}{ll}\text { - } & \text { SBRT } \\
\text { - } & \text { Hypofractionation (45 Gy) }\end{array}$ \\
\hline Median OS (months) & 41.2 & Not powered \\
\hline
\end{tabular}

LRT, locoregional therapy; PFS, progression-free survival; OS, overall survival; SBRT, stereotactic body radiotherapy; SOM-NSCLC, synchronous oligometastatic non-small lung cell cancer; ALK, anaplastic lymphoma kinase; EGFR, epidermal growth factor receptor; TKIs, tyrosine kinase inhibitors.

observed using LRT for SOM-NSCLC from two important prospective clinical trials summarized in Table 1.

In the first study by Gomez et al., 49 patients with good performance status i.e., Eastern Cooperative Oncology Group performance status score of $\leq 2$ and $\leq 3$ metastases received first line systemic therapy (platinum-based doublet) for 4-6 cycles. For patients with tumor driven mutations, 3 -month of tyrosine kinase inhibitors (TKIs) targeting epidermal growth factor receptor (EGFR) mutations or anaplastic lymphoma kinase (ALK) gene rearrangement were used. Randomization to maintenance systemic therapy with or without LRT (surgery or radiation) was offered to patients with no disease progression after the first line. The median progression-free survival (PFS) in the LRT group was 11.9 vs. 3.9 months in the maintenance treatment group [hazard ratio 0.35 (90\% CI, 0.18-0.66), $\log$-rank $\mathrm{P}=0.0054]$. Updates of survival data at 3 -year showed a durable improvement of PFS and OS in LRT arm compared to the control arm (14.2 vs. 4.4 months, $\mathrm{P}=0.022)$ and (41.2 vs. 17.0 months, $\mathrm{P}=0.017)$, respectively (1). A continuous OS benefit was reported after disease progression for patients treated by ablative LRT (median 37.6 vs. 9.4 months, $\mathrm{P}=0.034$ ). In the same study, salvage LRT was still a feasible option for $41 \%$ of patients who progressed. In multivariate analysis, LCT trended to correlate with better OS when used initially or as salvage.
Twenty percent of patients experienced $\geq$ grade 3 toxicity in the LRT arm compared to $8.3 \%$ in the control group in this trial (1).

In another phase II randomized clinical trial, by Iyengar et al., 29 patients with oligometastatic NSCLC ( $\leq 5$ lesions) were randomized to consolidative SBRT to all lesions or continuation of conventional maintenance systemic therapy. The primary thoracic disease was treated by SBRT or hypofractionated radiation. SBRT single-fraction doses included 21 to 27 Gy. Three-fraction SBRT doses included 26.5 to 33.0 Gy. Five-fraction cumulative doses included 30.0 to 37.5 Gy. If SBRT fractionation schemes for primary disease did not allow normal tissue constraints to be met, an alternative hypofractionation protocol was $45 \mathrm{~Gy}$ in 15 fractions. Median PFS for the SBRT and chemotherapy arm was 9.7 months compared to 3.5 months in chemotherapy only arm $(\mathrm{P}=0.01)$ with comparable toxicity rates between both groups (2).

Although LRT in SOM disease achieves durable local control, distant failures are still of high concern. In Tree et al., $80 \%$ of distant progression of SOM disease after SBRT treatments occurred within 2-4 years (20). Median time to progression from the two NSCLC trials ranged from (9-11 months) $(1,2)$. For patients presenting with oligometastatic recurrent lesions after initial LRT, salvage LRT could still be offered as a salvage treatment option, 
although there is no clear data about time sequencing of LRT and systemic therapies.

We are observing a major shift towards incorporating immunotherapy as early as possible in the course of management of NSCLC patients. The PD-L1 inhibitor, durvalumab has become the standard of care as consolidative therapy for Stage III NSCLC after completion of definitive concurrent chemoradiotherapy (21). For stage IV NSCLC, ICB has demonstrated improved median OS and PFS in the first-line as well as second-line settings in both squamous and non-squamous NSCLC (22-24). Synergy is expected when oligometastatic lesions are treated with LRT, especially SBRT, in combination with ICB. In a Phase II prospective clinical trial, pembrolizumab was used after 4 to 12 weeks of completing LRT to 51 OM-NSCLC patients who had $\leq 4$ metastatic sites. LRT arm was associated with a median PFS of 19.1 months, significantly greater than the historical median of 6.6 months $(\mathrm{P}=0.005)$. Median PFS was 18.7 months (95\% CI, 10.1-27.1 months) when maintenance pembrolizumab was added (25). Phase III trials are ongoing to further investigate the synergistic role of ICB in SOM-NSCLC. For example, the LONESTAR trial (NCT03391869) is a Phase III trial currently recruiting metastatic NSCLC patients to evaluate LRT value after ICB compared to ICB alone. This trial offers nivolumab and ipilimumab in combination with local consolidative therapy with either surgery or radiation (26).

Different phenotypes and genotypes carry a predictive value to LRT in SOM. Oncogene-driven tumors tended to benefit from LRT with relevant TKIs in first and 2nd line $(16,27)$, which will be elucidated further by the ongoing clinical trial (NCT03410043) that is treating EGFRmutated patients with Osimertinib and LRT. The results of the meta-analysis by $\mathrm{Li}$ et al., showed that having an EGFR mutation is an important predictive factor linked to improved OS for NSCLC patients with brain metastases (28). In a retrospective study by Weickhardt et al., patients who had oligoprogression on their first TKI erlotinib or crizotinib showed improved median progression free survival when LRT was combined with the same line of targeted therapy supporting the value of SBRT as an effective modality controlling these sites that may have lost its oncogenic addictive response and delaying the switch to second line TKI's (16). Similar improvement was reported when LRT for oligometastatic and primary sites was added to first line EGFR-TKI in SOM-NSCLC retrospective analysis by $\mathrm{Xu}$ et al. (27).
In a prospective single arm analysis of 24 patients with extra cranial oligoprogression, erlotinib was combined with SBRT to treat oligometastatic sites progressed after $1^{\text {st }}$ line chemotherapy. Median PFS was 14.7 months, and median OS was 20.4 months with SBRT with only $6 \%$ of failure at the irradiated sites (29). Results of $2^{\text {nd }}$ generation of EGFR TKI osimertinib are promising with clinically meaningful efficacy against CNS metastases with a high disease control rate and durable response regardless of radiation therapy (30). Randomizing SOM-NSCLC patients to more effective TKIs as osimertinib with LRT is expected to answer two questions; first if that local ablative treatments may add toxicity in a good prognostic group, or if local treatment will enhance these improved outcomes when combined to the TKI therapy. Ongoing trials such as NORTHSTAR (NCT03410043) attempts to address these points (31).

The intrinsic molecular subtypes of colorectal cancer with liver oligometastatic lesions were able to differentiate three robust risk groups based on gene expression profiling. This molecular analysis helps predict survival outcomes with LRT and identify patients with curable liver oligometastasis (32). Similar profiles for SOM NSCLC are awaited to further clarify tumors with limited metastatic capability where LRT is of maximum value. Circulating tumor DNA (ctDNA) mutational burden was reported as a quantitative biomarker that correlated with NSCLC evolution and proposed to identify an early relapsing or metastatic stage (33). In a correlative study (34), ctDNA metrics, cytokine levels, and T-cell receptor biology were studied in association to LRT in oligometastatic NSCLC treated within phase II trial (1). These biological markers are expected to guide patient selection for future definitive LRT trials.

Although the EORTC consensus on SOM-NSCLC was a step to define this evolving disease stage, additional evidence is still needed. Ongoing prospective randomized clinical trials summarized in Table 2 are recruiting to consolidate the role of LRT in SOM-NSLC stage with integration of immunotherapy and targeted therapies. Finally, it is emphasized that consensus on appropriate treatment of SOM-NSCLC patients in our current clinical practice should be obtained within dedicated thoracic multidisciplinary discussions addressing risks and benefits in view of the current evidence.

\section{Acknowledgments}

Funding: None. 
Table 2 Ongoing studies on LRT of SOM-NSCLC patients

\begin{tabular}{lcccc}
\hline Clinicaltrials.gov identification number & Phase & Number of patients & Primary end point & Country \\
\hline NCT03137771 (NRG trial-LU002) & II/III & 300 & PFS/OS & OS/PFS \\
NCT02417662 (SARON) & III & 340 & PFS & UK \\
NCT03119519 & II & 148 & OS & OS \\
NCT03827577 (OMEGA) & III & 195 & Italy \\
NCT03391869 (MDACC) & III & 270 & PFS USA & Europe, multicenter \\
NCT03965468 (CHESS) & II & 47 & PFS & China \\
NCT02893332 (sindas) & III & II & 142 & PFS \\
NCT03808337 (promise-005)
\end{tabular}

*, recruiting both breast and NSCLC oligometastatic patients. PFS, progression-free survival; OS, overall survival; LRT, locoregional therapy; SOM-NSCLC, synchronous oligometastatic non-small lung cell cancer.

\section{Footnote}

Provenance and Peer Review: This is an invited article commissioned and reviewed by the Section Editor Xiaozheng Kang (Department of Thoracic Surgery, Beijing Cancer Hospital, Peking University, Beijing, China).

Conflicts of Interest: All authors have completed the ICMJE uniform disclosure form (available at http://dx.doi. org/10.21037/jtd-20-1485). MP reports personal/consulting fees from Bayer Corporation, outside the submitted work; SKJ reports grants and personal fees from Merck, outside the submitted work. The other authors have no conflicts of interest to declare.

Ethical Statement: The authors are accountable for all aspects of the work in ensuring that questions related to the accuracy or integrity of any part of the work are appropriately investigated and resolved.

Open Access Statement: This is an Open Access article distributed in accordance with the Creative Commons Attribution-NonCommercial-NoDerivs 4.0 International License (CC BY-NC-ND 4.0), which permits the noncommercial replication and distribution of the article with the strict proviso that no changes or edits are made and the original work is properly cited (including links to both the formal publication through the relevant DOI and the license). See: https://creativecommons.org/licenses/by-nc-nd/4.0/.

\section{References}

1. Gomez DR, Tang C, Zhang J, et al. Local consolidative therapy vs. Maintenance therapy or observation for patients with oligometastatic non-small-cell lung cancer: Long-term results of a multi-institutional, phase II, randomized study. J Clin Oncol 2019;37:1558-65.

2. Iyengar P, Wardak Z, Gerber DE, et al. Consolidative radiotherapy for limited metastatic non-small-cell lung cancer: A phase 2 randomized clinical trial. JAMA Oncol 2018;4:e173501.

3. Hellman S, Weichselbaum RR. Oligometastases. J Clin Oncol 1995;13:8-10.

4. Niibe Y, Hayakawa K. Oligometastases and oligorecurrence: The new era of cancer therapy. Jpn J Clin Oncol 2010;40:107-11.

5. Weichselbaum RR, Hellman S. Oligometastases revisited. Nat Rev Clin Oncol 2011;8:378-82.

6. Dingemans AC, Hendriks LEL, Berghmans T, et al. Definition of Synchronous Oligometastatic Non-Small Cell Lung Cancer-A Consensus Report. J Thorac Oncol 2019;14:2109-19.

7. Giaj-Levra N, Giaj-Levra M, Durieux V, et al. Defining Synchronous Oligometastatic Non-Small Cell Lung Cancer: A Systematic Review. J Thorac Oncol 2019;14:2053-61.

8. Eberhardt WEE, Mitchell A, Crowley J, et al. The IASLC lung cancer staging project: Proposals for the revision of the $M$ descriptors in the forthcoming eighth edition of 
the TNM classification of lung cancer. J Thorac Oncol 2015;10:1515-22.

9. Ashworth AB, Senan S, Palma DA, et al. An individual patient data metaanalysis of outcomes and prognostic factors after treatment of oligometastatic non-small-cell lung cancer. Clin Lung Cancer 2014;15:346-55.

10. Clinical prognostic factors in surgically treated stage IIIA-N2 non-small cell lung cancer: analysis of the literature. Elsevier [Internet]. [cited 2020 Feb 20]. Available online: https://www.sciencedirect.com/science/ article/pii/S016950029700072X

11. De Ruysscher D, Wanders R, Van Baardwijk A, et al. Radical treatment of non-small-cell lung cancer patients with synchronous oligometastases: Long-term results of a prospective phase II trial (Nct01282450). J Thorac Oncol 2012;7:1547-55.

12. Darling GE, Dickie AJ, Malthaner RA, et al. Invasive mediastinal staging of non-small-cell lung cancer: A clinical practice guideline. Curr Oncol 2011;18:e304.

13. Collen C, Christian N, Schallier D, et al. Phase II study of stereotactic body radiotherapy to primary tumor and metastatic locations in oligometastatic nonsmall-cell lung cancer patients. Ann Oncol 2014;25:1954-9.

14. Gray PJ, Mak RH, Yeap BY, et al. Aggressive therapy for patients with non-small cell lung carcinoma and synchronous brain-only oligometastatic disease is associated with long-term survival. Lung Cancer 2014;85:239-44.

15. Yegya-Raman N, Aisner J, Kim S, et al. Synchronous Oligometastatic Non-small Cell Lung Cancer Managed With Curative-Intent Chemoradiation Therapy: Longterm Outcomes From a Single Institution. Adv Radiat Oncol 2019;4:541-50.

16. Weickhardt AJ, Scheier B, Burke JM, et al. Local ablative therapy of oligoprogressive disease prolongs disease control by tyrosine kinase inhibitors in oncogeneaddicted non-small-cell lung cancer. J Thorac Oncol 2012;7:1807-14.

17. Van den Begin R, Engels B, Collen C, et al. The METABANK score: A clinical tool to predict survival after stereotactic radiotherapy for oligometastatic disease. Radiother Oncol 2019;133:113-9.

18. Ashworth A, Rodrigues G, Boldt G, et al. Is there an oligometastatic state in non-small cell lung cancer? A systematic review of the literature. Lung Cancer 2013;82:197-203.

19. Jabbour SK, Daroui P, Moore D, et al. A novel paradigm in the treatment of oligometastatic non-small cell lung cancer. J Thorac Dis 2011;3:4-9.

20. Tree AC, Khoo VS, Eeles RA, et al. Stereotactic body radiotherapy for oligometastases. Lancet Oncol 2013;14:e28-37.

21. Antonia SJ, Villegas A, Daniel D, et al. Overall Survival with Durvalumab after Chemoradiotherapy in Stage III NSCLC. N Engl J Med 2018;379:2342-50.

22. Gandhi L, Rodríguez-Abreu D, Gadgeel S, et al. Pembrolizumab plus Chemotherapy in Metastatic NonSmall-Cell Lung Cancer. N Engl J Med 2018;378:2078-92.

23. Paz-Ares L, Luft A, Vicente D, et al. Pembrolizumab plus Chemotherapy for Squamous Non-Small-Cell Lung Cancer. N Engl J Med 2018;379:2040-51.

24. Papadimitrakopoulou V, Cobo M, Bordoni R, et al. OA05.07 IMpower132: PFS and Safety Results with 1L Atezolizumab + Carboplatin/Cisplatin + Pemetrexed in Stage IV Non-Squamous NSCLC. J Thorac Oncol 2018;13:S332-3.

25. Bauml JM, Mick R, Ciunci C, et al. Pembrolizumab after Completion of Locally Ablative Therapy for Oligometastatic Non-Small Cell Lung Cancer: A Phase 2 Trial. JAMA Oncol 2019;5:1283-90.

26. Phase III Trial of (LCT) After Nivolumab and Ipilimumab - Full Text View - ClinicalTrials.gov [Internet]. [cited 2020 Jan 5]. Available online: https://clinicaltrials.gov/ct2/show/ NCT03391869

27. Xu Q, Zhou F, Liu H, et al. Consolidative Local Ablative Therapy Improves the Survival of Patients With Synchronous Oligometastatic NSCLC Harboring EGFR Activating Mutation Treated With First-Line EGFRTKIs. J Thorac Oncol 2018;13:1383-92.

28. Li WY, Zhao TT, Xu HM, et al. The role of EGFR mutation as a prognostic factor in survival after diagnosis of brain metastasis in non-small cell lung cancer: a systematic review and meta-analysis. BMC Cancer 2019;19:145.

29. Iyengar P, Kavanagh BD, Wardak Z, et al. Phase II trial of stereotactic body radiation therapy combined with erlotinib for patients with limited but progressive metastatic non-small-cell lung cancer. J Clin Oncol 2014;32:3824-30.

30. Goss G, Tsai CM, Shepherd FA, et al. CNS response to osimertinib in patients with T790M-positive advanced NSCLC: Pooled data from two phase II trials. Ann Oncol 2018;29:687-93.

31. Elamin YY, Antonoff M, Blakely C, et al. 1509TiPRandomized phase II trial of osimertinib with or without local consolidation therapy (LCT) 
for patients with EGFR-mutant metastatic NSCLC (NORTHSTAR). Ann Oncol [Internet]. 2018 Oct 1 [cited 2020 Jan 5];29(suppl_8). Available online: https:// academic.oup.com/annonc/article/doi/10.1093/annonc/ mdy292.130/5140809

32. Pitroda SP, Khodarev NN, Huang L, et al. Integrated molecular subtyping defines a curable oligometastatic state in colorectal liver metastasis. Nat Commun 2018;9:1793.

33. Abbosh C, Birkbak NJ, Wilson GA, et al. Phylogenetic

Cite this article as: Almeldin DS, Malhotra J, Patel M, Aisner J, Jabbour SK. Local treatment of synchronous oligometastatic non-small cell lung cancer (NSCLC) - current consensus and future perspectives. J Thorac Dis 2020;12(11):7069-7075. doi: $10.21037 /$ jtd-20-1485
ctDNA analysis depicts early-stage lung cancer evolution. Nature 2017;545:446-51.

34. Tang C, Lee WC, Reuben A, et al. Immune and Circulating Tumor DNA Profiling After Radiation Treatment for Oligometastatic Non-Small Cell Lung Cancer: Translational Correlatives from a Mature Randomized Phase II Trial. Int J Radiat Oncol Biol Phys 2020;106:349-57. 